\title{
AVALIAÇÃO DA RECUPERAÇÃO DOS METAIS PRATA, CHUMBO E ZINCO PRESENTES EM REJEITO DE PROCESSAMENTO HIDROMETALÚRGICO DE ZINCO
}

\author{
J. OSTI', M. M. LOPES, F. C. NOGUEIRA, C. A. PEREIRA \\ Universidade Federal de Ouro Preto \\ jadeosti.0@gmail.com*
}

Submetido 11/10/2017 - Aceito 11/11/2017

DOI: $10.15628 /$ holos.2017.6393

\begin{abstract}
RESUMO
Com o desenvolvimento de tecnologias e uma crescente demanda por recursos minerais, a otimização da flotação ganhou importância como forma de aproveitar os recursos e reprocessar rejeitos que ainda contém metais de interesse. A viabilidade desta técnica depende do uso de reagentes químicos, que induzem condições de seletividade, hidrofobicidade e eficiência do processo. Esse trabalho tem por objetivo caracterizar e estudar formas de recuperação do rejeito de processamento hidrometalúrgico de zinco, da barragem de Murici da unidade da Votorantim Metais, localizada em Três Marias - MG. Por se tratar de um rejeito hidrometalúrgico sabe-se que esse tipo resíduo é complexo devido às transformações físico-químicas ao qual foi submetido e, portanto há pouca pesquisa desenvolvida nessa área. Dessa forma, esse trabalho contempla ensaios de caracterização do rejeito, como: análise granulométrica, testes de difração de raios $X$, análise química, de densidade e de separação magnética, e ensaios de concentração por flotação.
\end{abstract}

Os ensaios de flotação foram feitos com condicionamento em alta intensidade em escala de laboratório, sendo também estudado os diferentes reagentes (dispersantes e coletores) e suas dosagens, que possam consequentemente recuperar os metais de maior interesse ( $\mathrm{Ag}, \mathrm{Pb}$ e $\mathrm{Zn})$, desenvolvendo conhecimento científico e soluções para a empresa Votorantim. Os resultados de caracterização mostraram que $80 \%$ do material é menor que $100 \mu \mathrm{m}$, e que $50 \%$ está abaixo de $37 \mu \mathrm{m}$. Os minerais identificados na difração de raio $x$ foram: gesso, quartzo, bassanita, hematita, muscovita, caulinita, jarosita (plumbo) e calcita. Alguns resultados da flotação mostraram esse pode ser um mecanismo de aproveitamento dos rejeitos, uma vez que neste estudo obteve-se certa recuperação de $\mathrm{Pb}$ e $\mathrm{Zn}$ com o uso dos reagentes: silicato de sódio como depressor, sulfeto de sódio como ativador, Aeroine 3418A promoter + Amil xantato como coletores, Mibcol como espumante e um $\mathrm{pH}$ final de 9,46.

PALAVRAS-CHAVE: rejeito hidrometalúrgico, flotação, condicionamento em alta intensidade.

\section{EVALUATION OF SILVER, LEAD AND ZINC RECOVERY PRESENT IN A HYDROMETALLURGY TAILINGS}

\section{ABSTRACT}

With the development of new technologies and an increasing demand for mineral resources, the optimization of flotation has got importance as a way to utilize the mineral resources and reprocess waste that still contains metals of interest. The viability of this technique depends on the use of chemical reagents, which induce conditions of selectivity, hydrophobicity and process efficiency. This research aims to characterize and study ways to recover the residue from hydrometallurgical processing of zinc, from the Murici dam of Votorantim Metais unit, located in Três Marias - MG. The hydrometallurgical waste it is known as a complex type of residue, due to the physical-chemical transformations that this one was submitted and there is just a little research developed in this area. In this way, this work includes tests of characterization of the tailings, such as granulometric analysis, $\mathrm{X}$-ray diffraction tests, chemical analysis, density and magnetic separation, and flotation concentration tests. The flotation
\end{abstract}

tests were done with high intensity conditioning in laboratory scale, and it was also studied the different reagents (dispersants and collectors) and their dosages, which can consequently recover the metals of greater interest $(\mathrm{Ag}, \mathrm{Pb}$ and $\mathrm{Zn}$ ), developing scientific knowledge and solutions for Votorantim Metais. The characterization results showed that $80 \%$ of the material is smaller than $100 \mu \mathrm{m}$, and that $50 \%$ is below of $37 \mu \mathrm{m}$. The minerals identified in x-ray diffraction were: gypsum, quartz, bassanite, hematite, muscovite, kaolinite, jarosite (lead) and calcite. Some results showed that flotation can be a mechanism of utilization of waste, since in this study a certain recovery of $\mathrm{Pb}$ and $\mathrm{Zn}$ was obtained with the use of the reagents: Sodium silicate as depressant, sodium sulfide as activator, Aerophine $3418 \mathrm{~A}$ promoter + Amil xanthate as collectors, Mibcol as foaming agent and a final $\mathrm{pH}$ of 9.46 .

KEYWORDS: hydrometallurgical reject, floating, conditioning in high intensity. 


\section{INTRODUÇÃO}

A flotação é um processo muito difundido para concentração de minerais, sendo um processo dependente das diferenciabilidade das características na superfície das várias espécies minerais presentes no sistema. A viabilidade desta técnica depende do uso de reagentes químicos, utilizados para induzir as condições ideais de seletividade e eficiência do processo (WILLS, 2007).

Considerando que há uma crescente demanda por recursos minerais e que estes recursos estão cada vez mais complexos para serem tratados, a otimização da flotação tem ganhado a importância, com o desenvolvimento de novas tecnologias e equipamentos. As variáveis que influenciam este eficiência são: a distribuição de tamanho das partículas, a carga superficial, a hidrofobicidade, a hidrodinâmica do sistema, o condicionamento, pH e aeração da polpa (BULATOVIC, 2007).

Ainda segundo Bulatovic (2007), o grande avanço na utilização da flotação pela indústria mineral ocorreu a partir da introdução dos xantatos na flotação seletiva dos sulfetos. Atualmente, aproximadamente $95 \%$ da produção mundial de cobre, níquel, chumbo e zinco é obtida através deste processo. É comum utilizar a combinação de xantatos, mercaptantes e/ou ditiofosfatos.

Minérios de chumbo e zinco são um dos minérios mais abundantes do mundo. A maior parte do minério de chumbo-zinco apresenta também prata e até ouro. Quase $85 \%$ da produção de prata no mundo vem de minérios de chumbo-zinco. 0 sistema de reagentes utilizados na flotação no tratamento de minérios de chumbo-zinco varia consideravelmente dependendo da natureza e da mineralogia do minério. Na maioria dos casos, o sistema de depressor cianeto de sódio e sulfato de zinco é utilizado com xantato e ditiofosfato como coletores. No entanto, para tratamento de minérios sulfetados, um esquema de reagente muito mais complexo é utilizado (BULATOVIC, 2007).

A empresa Votorantim Metais Zinco S/A, é uma das empresas produtoras de zinco no mundo, no Brasil possui empreendimentos mineiros em Vazante em Paracatu, e ainda duas usinas hidrometalúrgicas, uma em Três Marias e uma em Juiz de Fora. Em Três Marias são tratados simultaneamente os concentrados de zinco sulfetados da mina de Morro Agudo e os concentrados silicatados de zinco da mina de Vazante. Nesse processo é gerado um rejeito hidrometalúrgico (SANTOS, 2009).

Com a depleção das reservas minerais, buscar alternativas para o aproveitamento de minérios com teores cada vez mais baixos tem sido um grande desafio para mineração. $O$ reaproveitamento de rejeitos também vem sendo bastante estudado pelas mineradoras pois além de gerar um produto com valor agregado, é possível reduzir ambiental, contribuindo para uma mineração sustentável.

Esse trabalho tem por objetivo caracterizar e através de testes de exploratórios de flotação estudar formas de recuperação do rejeito hidrometalúrgico de zinco, da barragem de Murici da unidade da Votorantim Metais, localizada em Três Marias - MG. 


\section{METODOLOGIA}

A parte experimental desse projeto foi realizada nos Laboratórios de Tratamento de Minérios do Departamento de Engenharia de Minas da Universidade Federal de Ouro Preto. A caracterização mineralógica foi realizada no laboratório de Difração de Raios $X$ da Universidade Federal de Minas Gerais e as análises químicas foram feitas na empresa Votorantim Metais, unidade de Vazante.

\subsection{Preparação das amostras de rejeito}

A Votorantim Metais - Unidade de Três Marias coletou e disponibilizou amostra do rejeito do processo hidrometalúrgico de zinco, da barragem de Murici. A massa total do rejeito enviado para a universidade foi igual à 390kg.Para assegurar a representatividade do material amostrado, foram realizadas as seguintes etapas:

i. A amostra inicial de $390 \mathrm{~kg}$ foi homogeneizada e dividida primeiramente em um quarteador carrossel contendo doze recipientes. Foram realizados quatro quarteamentos carrossel com abertura de $3 \mathrm{~cm}$. De cada quarteamento foram selecionadas 2 alíquotas por sorteio, formando assim um conjunto de 8 alíquotas;

ii. Essas 8 alíquotas foram homogeneizadas e submetidas ao quarteador carrossel novamente. Nessa segunda fase de quarteamento no carrossel foram selecionadas novamente duas alíquotas por sorteio;

iii. As duas alíquotas foram divididas no quarteador Jones e compostas até formarem amostras representativas de $381,5 \mathrm{~g}$.

\subsection{Caracterização do rejeito hidrometalúrgico}

Para caracterizar o rejeito foram feitas análises granulométrica, testes de difração de raios $X$, análise química, de densidade, de separação magnética e por caracterização mineralógica. As análises químicas foram realizadas pela ALS Minerals, com uma parceria com a empresa Votorantim.

\subsection{Testes de flotação}

Para os testes utilizada uma máquina de flotação CDC, modelo GFB - 1000 EEPNBA e uma cuba de acrílico com capacidade de 3,6 litros, dotada de um sistema de raspagem automática de espuma e de um impelidor de disco central e pás verticais que geraram um fluxo predominantemente radial. A polpa possuía $10 \%$ de sólido, e a etapa de condicionamento dos reagentes foi feita em alta turbulência (1800-2200rpm).

Primeiramente foi adicionado 1,3L e 381,5g de rejeito. Em seguida a polpa era transferida para a cuba e adicionada mais $1,7 \mathrm{~L}$ de água. $\mathrm{O}$ pH era ajustado para próximo de 9,5, em seguida era adicionado depressor e condicionado por 6 minutos.

Após isso era incluído o ativador e condicionado por mais 6 minutos. A pós esse tempo a velocidade de agitação era elevada (entre $1800-2200 \mathrm{rpm}$ ) e adicionada a solução de coletores, que era condicionada por mais 10 minutos. Finalmente acrescentava-se o espumante MIBCOL e a polpa era condicionada por 2 minutos. 
Após esse condicionamento a velocidade de rotação era diminuída para $1200 \mathrm{rpm}$, a válvula de ar aberta e coletava-se espuma por 3 minutos. Os produtos desses testes eram filtrados, secados e enviados para análises.

Os reagentes usados estão na Tabela 1 abaixo, foi usado 2000g/t de depressor, $1000 \mathrm{~g} / \mathrm{t}$ de ativador e $150 \mathrm{~g} / \mathrm{t}$ de coletor.

Tabela 1. Reagentes utilizados nos testes.

\begin{tabular}{ll}
\hline Função & Reagente \\
\hline Modificador de $\mathrm{pH}$ & $\begin{array}{l}\text { Ácido clorídrico } \\
\text { Hidróxido de Sódio }\end{array}$ \\
\hline Depressor & Silicato de Sódio \\
\hline Ativador & Sulfeto de Sódio \\
\hline Coletor & Amil Xantato \\
\hline & AEROFLOAT 242 \\
& AERO 7376 promoter \\
& AEROPHINE 3418A promoter \\
& AERO 407 \\
& AERO MX 6222 \\
& FLOTIGAM2835 2L \\
& FLOTIGAM EDA \\
\hline Espumante & Mibcol \\
\hline
\end{tabular}

Ao todo foram feitos 14 testes de flotação em bancada, os coletores foram usados individualmente e combinados entre si, como: Amil Xantato+AEROFLOAT 242, Amil Xantato+AERO 7376 promoter, Amil Xantato+AEROPHINE 3418A promoter, Amil Xantato+AERO 407, Amil Xantato +AERO MX 6222, Amil Xantato+FLOTIGAM 2835 2L, Amil Xantato+FLOTIGAM EDA.

\section{RESULTADOS E DISCUSSÕES}

\subsection{Caracterização do rejeito hidrometalúrgico}

Pelas análises granulométricas pode-se afirmar que $80 \%$ do material se encontra abaixo de $100 \mu \mathrm{m}$, e que $50 \%$ de todo o material está com granulometria menor que $37 \mu \mathrm{m}$.

A análise de difração de raio $x$ foi feita com duas amostras, uma antes da separação magnética e uma após este processo. Na análise antes da separação magnética foram identificados os seguintes minerais: gesso, quartzo e bassanita. Após a separação foram identificados: quartzo, hematita, muscovita, caulinita, jarosita (plumbo) e calcita.A picnometria resultou numa densidade média de 2,31. 


\subsection{Testes de flotação de bancada}

Os resultados obtidos para recuperação de $\mathrm{Pb}, \mathrm{Zn}$ e $\mathrm{Ag}$ estão apresentados na tabela seguinte (Tabela 2). Em todos os ensaios foi utilizado silicato de sódio como depressor e sulfeto de sódio ativador.

Tabela 2. Resultados obtidos nos testes de flotação

\begin{tabular}{|c|c|c|c|c|c|c|c|}
\hline \multicolumn{2}{|c|}{ TESTES } & COLETOR & \multirow{2}{*}{$\begin{array}{l}\text { ESPUMANTE } \\
\text { Mibcol }\end{array}$} & \multirow{2}{*}{$\begin{array}{r}\mathbf{p H} \\
9,64\end{array}$} & \multirow{2}{*}{$\begin{array}{c}\begin{array}{c}\text { Rec. Ag } \\
\text { (ppm) }\end{array} \\
182,52\end{array}$} & \multirow{2}{*}{$\begin{array}{l}\begin{array}{c}\text { Rec. Pb } \\
(\% 0,0002)\end{array} \\
1,05\end{array}$} & \multirow{2}{*}{$\begin{array}{l}\text { Rec. Zn } \\
(\% 0,01)\end{array}$} \\
\hline Flotado & $\mathrm{T} 1$ & Aerofloat $242+$ Amil & & & & & \\
\hline Afundado & & xantato & & 9,64 & 151,58 & 0,96 & 2,87 \\
\hline Flotado & $\mathrm{T} 2$ & Aerofloat 242 & Mibcol & 9,55 & 222,91 & 1,08 & 6,3 \\
\hline Afundado & & & & 9,55 & 167,1 & 0,99 & 5,26 \\
\hline Flotado & T3 & Aero 7376 promoter + & Mibcol & 9,47 & 236,18 & 1,13 & 5,85 \\
\hline Afundado & & Amil xantato & & 9,47 & 256,75 & 1,22 & 6,43 \\
\hline Flotado & $\mathrm{T} 4$ & Aero 7376 promoter & Mibcol & 9,59 & 147,47 & 0,88 & 4,82 \\
\hline Afundado & & & & 9,59 & 203,28 & 1,12 & 5,9 \\
\hline Flotado & T5 & Aerophine & Mibcol & 9,43 & 226,5 & 1,14 & 6,52 \\
\hline Afundado & & promoter & & 9,43 & 263,37 & 1,5 & 6,47 \\
\hline Flotado & T6 & Aerophine & Mibcol & 9,46 & 327,07 & 1,69 & 7,95 \\
\hline Afundado & & $\begin{array}{l}\text { promoter }+ \text { Amil } \\
\text { xantato }\end{array}$ & & 9,46 & 127,08 & 0,71 & 5,46 \\
\hline Flotado & $\mathrm{T7}$ & Aero 407 & Mibcol & 9,56 & 176,9 & 0,88 & 5,87 \\
\hline Afundado & & & & 9,56 & 129,14 & 1,22 & 5,14 \\
\hline Flotado & T8 & Aero $407+$ Amil & Mibcol & 9,37 & 81,5 & 0,803 & 4,86 \\
\hline Afundado & & xantato & & 9,37 & 47,6 & 0,821 & 4,24 \\
\hline Flotado & T9 & Amil xantato & Mibcol & 9,5 & 48,2 & 0,799 & 4,37 \\
\hline Afundado & & & & 9,5 & 45,7 & 0,805 & 4,08 \\
\hline Flotado & $\mathrm{T} 10$ & Aero MX $6222+$ Amil & Mibcol & 9,54 & 46,5 & 0,786 & 3,55 \\
\hline Afundado & & xantato & & 9,54 & 44,1 & 0,792 & 3,93 \\
\hline Flotado & $\mathrm{T} 11$ & Aero MX 6222 & Mibcol & 9,54 & 45,6 & 0,812 & 4,08 \\
\hline Afundado & & & & 9,54 & 47,1 & 0,825 & 4,22 \\
\hline Flotado & T12 & Flotgam $28352 \mathrm{~L}+$ Amil & Mibcol & 9,62 & 44,8 & 0,82 & 4,06 \\
\hline Afundado & & xantato & & 9,62 & 43,5 & 0,816 & 4,45 \\
\hline Flotado & T13 & Flotgam $28352 \mathrm{~L}$ & Mibcol & 9,56 & 43,2 & 0,809 & 4,36 \\
\hline Afundado & & & & 9,56 & 50,7 & 0,801 & 4,11 \\
\hline Flotado & T14 & Flotgam EDA + Amil & Mibcol & 9,52 & 51,7 & 0,845 & 4,33 \\
\hline Afundado & & xantato & & 9,52 & 56,1 & 0,891 & 4,46 \\
\hline
\end{tabular}

A tabela indica o teor dos três metais de interesse, $\mathrm{Pb}, \mathrm{Zn}$ e $\mathrm{Ag}$, nas amostras de rejeito e concentrado dos testes exploratórios de flotação. O melhor conjunto de reagentes foi o do teste T6, que usou como reagentes: silicato de sódio, sulfeto de sódio, Aerophine 3418A promoter + Amil xantato, mibcol e apresentou um $\mathrm{pH}$ final de 9,46. 
Também é possível perceber que a partir do T8 os resultados não foram significativos, já que não houve aparente diferença entre os resultados de recuperação entre o flotado e afundado de cada teste.

\section{CONCLUSÕES}

É possível concluir com os resultados da caracterização que se trata de um rejeito de granulometria muito fina ( $80 \%$ abaixo de $100 \mu \mathrm{m}$ e $50 \%$ abaixo de $37 \mu \mathrm{m}$ ), sendo também muito complexo por ter passado por um processo hidrometalúrgico, e que apresenta metais de interesse para recuperação como $\mathrm{Pb}, \mathrm{Ag}$ e $\mathrm{Zn}$.

Com os resultados da flotação de bancada foi possível afirmar que o melhor conjunto de reagentes usados foi o do ensaio T6, que permitiu constituir um material flotado (oversize) com recuperações diferenciadas do afundado (undersize). Além disso foi o que obteve maiores recuperações de $\mathrm{Zn}, \mathrm{Pb}$ e Ag.

Segundo as necessidades da empresa, os resultados não alcançaram um valor significativo para a recuperação da prata, $>1000 \mathrm{ppm}$, porém considera-se como uma oportunidade de limpeza do chumbo e para intensificar novos estudos que possibilitem um efetivo aproveitamento deste rejeito.

\section{REFERÊNCIAS}

BULATOVIC, S.M. (2007). Handbook of Flotation Reagents: Chemistry, Theory and Practice. v. 1, Primeira Edição. Elsevier Science \& Tecnology Books. Amsterdam, p. 401- 441.

SANTOS, J. F. D. (2009). Relátorio Técnico 65 - Perfil do zinco. Ministério de Minas e Energia MME

SÃO JOSÉ, F. (2015). Flotação coletiva de sulfetos de zinco e chumbo. 2015. Dissertação (mestrado). Programa de Pós-Graduação em Engenharia Mineral. Universidade Federal de Ouro Preto, Ouro Preto.

WILLS, B.A., TIM NAPIER-MUNN (2006). Froth Flotation In: Mineral Processing Technology: An Introduction to the Practical Aspects of Ore Treatment and Mineral. Elsevier Science \& Technology Books. Sétima Edição. p. 267- 353. 